RESEARCH ARTICLE

\title{
Strength and Endurance Influence on the Trunk Muscle in the Functional Performance of Elderly Women
}

\section{Marceli Matos Andrade Mesquita1, Marta Silva Santos², Alan Bruno Silva Vasconcelos ${ }^{2}$, Antônio Gomes de Resende-Neto ${ }^{1}$, José Carlos Aragão-Santos², Roberto Jerônimo Santos Silva', Gilmar Weber Senna ${ }^{3}$, Felipe José Aidar Martins ${ }^{1,2}$, Paulo Márcio Pereira Oliveira ${ }^{4}$, Estélio Henrique Martin Dantas $^{5}$ and Marzo Edir Da Silva-Grigoletto ${ }^{1,2^{*}}$}

${ }^{1}$ Department of Physical Education, Federal University of Sergipe, Brazil

${ }^{2}$ Department of Physiology, Federal University of Sergipe, Brazil

${ }^{3}$ Nucleus of Physical Education and Sports, Federal University of the State of Rio de Janeiro, Brazil

${ }^{4}$ Department of Physiotherapy, Federal University of Sergipe, Brazil

${ }^{5}$ Tiradentes University, Brazil

*Corresponding author: Marzo Edir Da Silva-Grigoletto, Center of Biological and Health Sciences, Department of Physical Education, Federal University of Sergipe, Cidade Universitária Prof. José Aloísio de Campos, Av. Marechal Rondon, s/n Jardim Rosa Elze, 49100-000, São Cristóvão, Sergipe, Brazil

\begin{abstract}
Background: The trunk muscles have an important role in the stabilization of the trunk in the elderly, however it is not known how much they can influence in the functional performance of this specific population.
\end{abstract}

Objective: To verify the influence of strength and endurance on the trunk muscles on functional performance in elderly women.

Methods: There were 45 elderly women in the study. The maximum strength and endurance of the trunk and the functional performance were evaluated through the stable wooden seat, McGill protocol, Senior Fitness Test battery and the deadlift. For statistical purposes, a multiple regression model was used. The trunk strength and endurance were used as independent variables and functional performance as a dependent variable.

Results: There was a participation of trunk extensor muscles endurance from 17.9 to $24.4 \%$ in functional performance in the elderly. The maximum strength of these muscles did not influence the performance.

Conclusion: Strength and endurance have a significant influence on the trunk muscles on the measures of functional performance in elderly women.

\section{Keywords}

Performance, Stability, Aging, Dynapenia, Trunk muscle

\section{Abbreviations}

ADLs: Activities of daily life; N: Newton; SDR: Strength Development Rate

\section{Introduction}

The aging-related implications, such as decreased skeletal muscle thickness, are often accompanied by reduced muscle strength and functionality $[1,2]$. These muscular and functional changes are associated with a reduction in quality of life and an increased risk of falls among the elderly population [3]. This growth is a major health concern because it generates a growing socioeconomic burden due to the increase of injury, disability and mortality rates [4].

Studies have already demonstrated a strong relationship between the strength of the muscles of the lower limbs and the maintenance of postural stability in the elderly people $[5,6]$. Differently, less evidence is available about the importance of the muscles that 
stabilize the trunk and its relation with the movement of the extremities during the accomplishment of functional tasks [7]. Therefore, recent research has investigated the changes related to trunk musculature in the elderly people and their association with better performance during Activities of Daily Life (ADLs) and prevention of falls [3,8,9].

Muscle strength is an important physical ability evaluating the health status of an individual [10]. Trunk strength is associated with the production and transference of strength between the upper and lower limbs during the execution of complex multi-articular movements; besides reducing mechanical stress in the spine $[7,11,12]$. This can be considered very important for the elderly, as it would contribute to improve the ability to generate strength with lower limbs [13]. Regarding the changes in trunk muscle thickness over the years, reductions $26-48 \%$ of thickness were observed in the elderly $\geq 75$-years-old, when compared to young people between 30-50 years-old [2]. Hicks, et al. [11] also revealed associations between increased fat infiltration in the trunk muscles and reduced functional capacity and balance in the elderly over 70 -years-old.

According to the systematic review of Granacher, et al. [9] they identified associations between trunk muscle strength/increased fat infiltration and balance, as well as functional capacity and risk of falls in older adults. However, a high level of heterogeneity was observed in the results of the studies due to the presence of different methodological designs used, and these authors recommended an additional evaluation to observe the influence of the strength of the trunk muscles and functional capacity of the trunk in adults older [9].

Thus, given the importance of the trunk muscles for functional performance, the scarcity in the literature regarding the subject and the absence of a specific and sensitive functional evaluation for this purpose in this population, the primary objective of this study was to verify the influence of the strength and endurance in the trunk muscles on functional performance in the elderly women over 60 -years-old. We hypothesize that the strength and endurance of these muscles will be positively associated with functional capacity in the elderly women.

\section{Materials and Methods}

\section{Study design}

This is a cross-sectional study of a session assessing the maximum strength and endurance of the trunk muscles in the elderly women and functional performance. For this physical evaluation, a stable wooden seat was used to measure maximum isometric strength. Also, two tests of the McGill protocol for the endurance of these muscle groups, and three Senior Battery Fitness tests were performed to evaluate functional perfor- mance in the elderly women.

\section{Participants}

There were 45 trained and without impediment elderly women recruited through digital media, posters, and pamphlets to participate in the evaluations. (1) Women over 60-year-old; (2) Absence of limiting back pain in the last year; (3) Absence of cardiac instability that could interfere during the test; (4) Ability to walk without an aid device; (5) Speak and understand the Portuguese language was included in the study. The absence for one of the evaluation sessions was established as exclusion criteria for the study.

Before the evaluation session, all the elderly women were instructed to read the informed consent form and those who agreed to participate signed the form before starting the study. This study was submitted to the Ethics Committee in Research with Human Beings of the University in accordance with the principles of the Helsinki Declaration (1964) and approved under the number 060568/2017. All the participants lived at their home, used the public health system and carried out common activities such as walking and domestic activities, independently. They underwent a medical consultation and physical evaluation to prove their suitability for the tests.

\section{Procedures}

The tests were performed in the same place and time, administered in the same order and supervised by the same researchers. Before the tests, the researchers adjusted the devices and instructed the participants about their body positioning. All participants wore sportswear and were instructed to give the maximum effort during the tests, motivated by clapping and phrases.

Initially, the elderly answered to an anamnesis with questions regarding sociodemographic aspects: age, gender, and address; health aspects: presence or absence of musculoskeletal diseases (lumbar spine); and use of medicines. To characterize the sample, body mass and height measurements were performed using an anthropometric scale (Welmy, R-110, São Paulo, Brazil).

\section{Evaluation of the maximum strength of the trunk muscles}

A stable wooden seat with adjustable support for hip and lower limbs was constructed according to the height of each individual to evaluate the maximum strength of the trunk muscles, isolating only the trunk muscles at the time of the test [14]. To normalize the height of the volunteers to the device, the seat was adjusted so the participants had their arms crossed in front of the thorax; slightly anterior slope of the pelvis to avoid compensatory activation of lower limbs; and the legs secured to the seat by a Velcro strap [7]. 


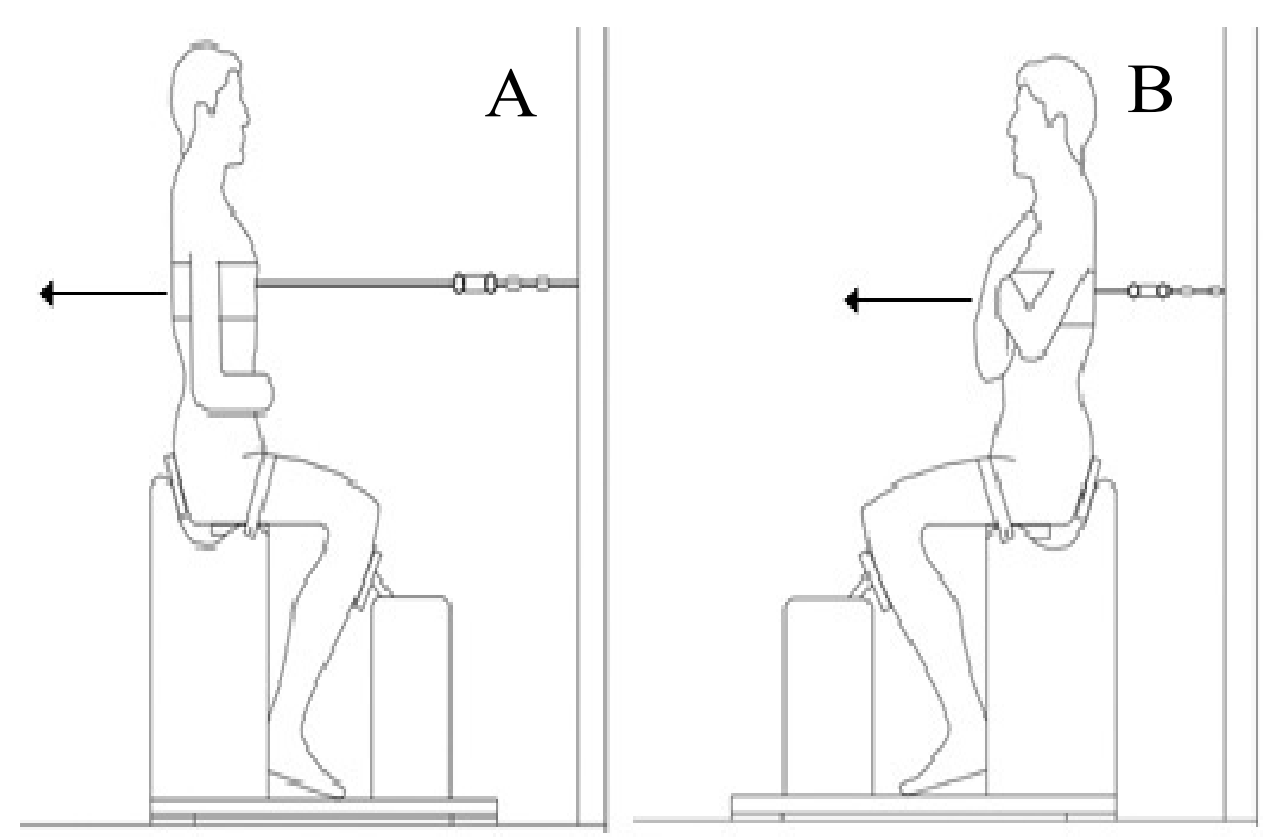

Figure 1: (A) Side view during trunk extension; (B) Side view during trunk flexion.

From this position, the muscle strength of the trunk extensors and flexors were measured through a digital load cell (Ktoyo, 333 A, Hown Dong, South Korea), connected to the Muscle Lab system (Ergotest Innovation, Porsgrunn, Norway) which provides a value in Newton (N), and the Strength Development Rate (SDR) in $N / s$.

In the evaluation of the trunk extensors, the participants were positioned with the trunk at $0^{\circ}$ of flexion (Figure 1A). The load cell was attached to the wall by an adjustable tensioner and connected to the individual by a Velcro strap at the level of the xiphoid process. From this positioning, a maximal isometric contraction in the trunk extension was requested. For the evaluation of the trunk flexors, the load cell was fixed to the wall behind the evaluated one, with the strap below the height of the scapula; and a maximal isometric contraction was requested in trunk flexion (Figure 1B).

For the measurement of strength in both muscles, the participants initially performed a maximal repetition to be familiarized with the test, followed by three maximum attempts, which lasted five seconds. Among them, the highest value was used for analysis purposes. The recovery intervals between maximal repetitions were 15 seconds and in all the attempts, both evaluators had the following verbal commands: "Prepare" (for the positioning of the individual); "Go, Strength, Strength, Strength, Relax" (for the test) [15].

\section{The endurance of the trunk muscles}

A timer (Technos, YP2151/8P, Rio de Janeiro, Brazil) was used to measure the maximum time the participants were able to maintain in each isometric position. Five minutes of recovery interval was established between each test [16]. For endurance assessment of the trunk flexors, participants were instructed to remain in the seated position with their backs resting on a wooden wedge and angle at $60^{\circ}$ from the ground. Both knees and hips were flexed $90^{\circ}$, arms crossed in front of the chest, hands on opposite shoulder and feet fixed. They were instructed to maintain the initial posture, while the wooden wedge was removed $10 \mathrm{~cm}$ in the posterior direction. The end of the test was determined when the upper trunk was unable to maintain the angle of $60^{\circ}$.

During the measurement of the endurance of the trunk extensors, participants were instructed to a position with the upper trunk over the edge of the litter at the height of the anterior superior iliac crest and with crossed arms in front of the chest. The pelvis, knees, and hip were fixed through three Velcro straps to keep the area assessed safe. For the beginning of the test, the command was given to maintain the horizontal position of the trunk as long as possible. The test was finished when the participant could no longer maintain the initial position [16].

\section{Functional performance}

The functional performance related to daily activities was evaluated through three tests of the Senior Battery Fitness test proposed by Rikli \& Jones [17], considering tests that evaluate the physiological capacity to perform normal daily activities independently.

a) Standing up and walking: It evaluates agility and dynamic balance. It began with the participant sitting in a chair, hands on her thighs and feet on the floor. At the evaluator's signal, the participant was standing up and walking as fast as possible, without running, skirting a cone at a distance of $2.44 \mathrm{~m}$, returning to the starting position. The shortest time was recorded after two attempts.

b) Sitting and standing up: It aims to evaluate the 
strength of the lower limbs. It started with the participant sitting on the chair and with her feet on the floor. Before beginning the test, the participant performed three repetitions to be familiarized with the task and then performed as many repetitions as possible for a period of 30 seconds.

c) Six-minute walking test: It evaluates cardiorespiratory endurance. The participant walked as fast as possible on a rectangular course $(45.72 \mathrm{~m}$ and demarcated by cones every $4.57 \mathrm{~m}$ ) for a period of 6 minutes to go as far as possible.

The lumbar dynamometer (Sammons Preston RoIyan, Jamar Dynamometer, hydraulic hand, Canada) was used to evaluate the maximum strength of the posterior trunk chain. Regarding the positioning of the test, participants were placed on a platform (standardized length), knees flexed at $100^{\circ}$ and hips slightly flexed. They were asked to perform maximum trunk extension strength with a gradual and constant movement, where there were two attempts and the highest value was considered [18].

\section{Statistical analysis}

Descriptive statistics were performed for the presentation of the data through the mean and standard

Table 1: Descriptive values of the strength and endurance variables of the trunk and functional performance of the elderly women.

\begin{tabular}{|l|l|}
\hline Variables & Mean and SD \\
\hline Maximal flexor strength $(\mathrm{N})$ & $234.61 \pm 66.47$ \\
\hline Maximal extensor strenght $(\mathrm{N})$ & $260.86 \pm 76.02$ \\
\hline Endurance of the flexor (s) & $71.53 \pm 66.01$ \\
\hline Endurance of the extensor (s) & $101.93 \pm 56.27$ \\
\hline Deadlift (kg/f) & $50.66 \pm 12.73$ \\
\hline Sitting and standing up (repetições) & $17.06 \pm 4.4$ \\
\hline Standing up and walking (s) & $4.65 \pm 0.53$ \\
\hline Walking for 6 minutes (m) & $544.80 \pm 58.88$ \\
\hline
\end{tabular}

deviation. The data distribution was tested by the Kolmogorov-Smirnov test. The trunk strength and endurance relationship with the functional performance tests were verified by linear regression models. The models were separated according to the independent variable category (strength and endurance) and flexor and extensor information were inserted simultaneously into the multiple and univariate models. Functional performance tests were used as dependent variables. The level of significance was set at $5 \%$. Statistical program SPSS 20.0 (SPSS Inc., Chicago, IL, USA) was used for all analyses.

\section{Results}

Forty-five trained and untrained elderly women participated in the study, with anthropometric characteristics: age $65.2 \pm 4.1$ years, stature $154.3 \pm 4.6 \mathrm{~m}$ and body weight $68.2 \pm 10.6 \mathrm{~kg}$. The strength and endurance variables of the trunk muscles and the functional performance are described in Table 1.

The multivariate regression using the stepwise type excluded the independent variables that did not influence the final model. Thus, in Table 2 and Table 3 , the values of $R^{2}$, its $p$-value and the individual beta $(\beta)$ are presented.

A significant result was demonstrated by a greater influence of trunk extensor endurance on the functional performance of active elderly women in all variables analyzed (sit and lift: $p<0.001$, lift and walk: $p<0.001$; $<$ $0.004)$, with the exception of the deadlift ( $p<0.788)$. Regarding maximum strength, only trunk flexors strength influenced the trunk posterior chain extension capacity, which was assessed by the deadlift $(p<0.001)$ Table 4 .

\section{Discussion}

Previous studies report that there is a production of the trunk muscles strength associated with the transfer of the strength between the upper and lower limbs during the execution of complex multi-articular

Table 2: Multivariate analysis of the influence of maximal strength of the trunk muscles on the functional performance variables in the elderly women.

\begin{tabular}{|l|l|l|l|}
\hline Variables & $\mathbf{R}^{\mathbf{2}}$ & Beta $(\boldsymbol{\beta})$ & p-value \\
\hline Deadlift (kg/f) & 0.275 & & $<0.001^{*}$ \\
\hline Maximal flexor strength (N) & & 0.014 & 0.524 \\
\hline Sitting and standing up (rep) & & -0.130 & 0.745 \\
\hline Maximal flexor strength (N) & & 0.145 & \\
\hline Maximal extensor strenght (N) & 0.033 & & 0.498 \\
\hline Standing up and walking (s) & & -0.111 & \\
\hline Maximal flexor strength (N) & & -0.088 & \\
\hline Maximal extensor strenght (N) & 0.010 & & 0.813 \\
\hline Walking for $\mathbf{6}$ minutes (m) & & 0.113 \\
\hline Maximal flexor strength (N) & & -0.120 \\
\hline Maximal extensor strenght (N) & & \\
\hline
\end{tabular}


Table 3: Multivariate analysis of the endurance influence of the trunk muscles on the functional performance variables in the elderly women.

\begin{tabular}{|c|c|c|c|}
\hline Variables & $\mathbf{R}^{2}$ & Beta $(\beta)$ & $p$-value \\
\hline Deadlift (kg/f) & \multirow[t]{3}{*}{0.012} & & \multirow{3}{*}{0.778} \\
\hline Endurance of the flexor (s) & & -0.067 & \\
\hline Endurance of the extensor (s) & & 0.140 & \\
\hline Sitting and standing up (rep) & \multirow[t]{2}{*}{0.244} & & \multirow[b]{2}{*}{$0.001^{*}$} \\
\hline Endurance of the extensor (s) & & 0.494 & \\
\hline Standing up and walking (s) & \multirow[t]{2}{*}{0.234} & & \multirow[b]{2}{*}{$0.001^{*}$} \\
\hline Endurance of the extensor (s) & & -0.484 & \\
\hline Walking for 6 minutes (m) & \multirow[t]{2}{*}{0.176} & & \multirow[b]{2}{*}{$0.004^{*}$} \\
\hline Endurance of the extensor (s) & & 0.420 & \\
\hline
\end{tabular}

Table 4: Univariate $\left(R^{2}\right)$ analysis of endurance influence and trunk muscle strength on the functional performance variables in the elderly women.

\begin{tabular}{|l|l|l|l|l|}
\hline Dependent variables & $\begin{array}{l}\text { Maximal flexor } \\
\text { strength (N) }\end{array}$ & $\begin{array}{l}\text { Maximal extensor } \\
\text { strength (N) }\end{array}$ & $\begin{array}{l}\text { Endurance of the } \\
\text { flexor (s) }\end{array}$ & $\begin{array}{l}\text { Endurance of the } \\
\text { extensor (s) }\end{array}$ \\
\hline Deadlift (Kg/f) & 0.275 & 0.127 & 0.001 & 0.009 \\
\hline Sitting and standing up (rep) & 0.001 & 0.004 & 0.180 & 0.244 \\
\hline Standing up and walking (s) & 0.028 & 0.025 & 0.061 & 0.234 \\
\hline Walking for 6 minutes (m) & 0.001 & 0.002 & 0.098 & 0.176 \\
\hline
\end{tabular}

movements [7,12]. However, this relationship is not yet well established in the literature, that is, it is uncertain, and a parameter of the trunk muscles actually influences more on functional performance in the elderly women. According to the data obtained in this study, it was observed that it is interesting that the endurance of the extensor muscles of the trunk influence more on the functional performance than the endurance of the flexors and the maximum strength. The findings of this study are aligned with our hypotheses, although the relationship between the peak strength and the performance measures was not as consistent as the relationship between endurance and functional performance.

The multivariate analyses between the maximum strength and the four measures performance revealed that only the deadlift obtained a significant relationship. It seems that isometric strength has not influenced the measures of functional ability, believing that the maximum strength of the trunk muscles is not necessary to perform simple tasks such as sitting and standing up, standing up and walking and walking for 6 minutes. Confirming our findings, a systematic review of Granacher, et al. [9] have observed that the associations between the standing up and walking tests and the Berg balance scale with maximum trunk strength is unclear and this is partly because the standing up and walking test requires multiple dimensions equilibrium and mobility, while the Berg balance scale comprises a series of static tasks that may be more related to trunk stability [9].

In contrast, in a multivariate analysis, Shahtahmasse- bi, et al. [19] observed small to moderate influence between maximal trunk muscle strength and measures of functional performance in the elderly people. However, it was a population that included men and women with a mean age of 70-years-old, which differs from our study [19]. By associating isometric strength and deadlift, there was a greater influence of the trunk flexor muscles than expected for this test, since, during its execution, there is a greater participation of the maximum strength of the extensor muscles of the trunk [18]. However, it could be explained due to the high requirement of the extensor muscles of the trunk during the deadlift, which could generate a co-activation of the trunk flexor muscles as a protective mechanism for the spine, activating deep trunk muscles, responsible for stabilization and maintenance of intra-abdominal pressure [20].

Multivariate analyses revealed a significant relationship between the endurance of the trunk extensor muscles and three functional performance tests (sitting and standing up, standing up and walking and walking for 6 minutes), except for the deadlift. Thus, it seems that the endurance of the extensor muscles of the trunk influences more on measures of functional performance. Suri, et al. [21] demonstrated that endurance in trunk extension was moderately correlated with the Berg balance scale $(r=0.41, p<0.05)$. The same authors suggested that a one-second increase in resistance to trunk extension was associated with a $4 \%$ increase in the probability of significant changes in the Berg balance scale, which acts positively in reducing falls in the elderly population [21]. Also, McGill, et al. [16] also emphasized the relative importance of endurance of the trunk muscles, which is consistent 
with the findings of this study.

The strength of the associations observed between the endurance of the trunk extensors and the performance results (sitting and standing up, standing up and walking and walking for 6 minutes) can be explained mechanically. The functional performance evaluation was composed of dynamic tasks and requiring variation of the support base to ensure stability. In tasks related to movement, and specifically those involving a forward slope in the sagittal plane, the involvement of the extensors of the trunk is greater, stabilizing the trunk [22]. Specifically, to the chair sitting and standing up test, there was a greater contribution to the endurance of the trunk extensors. Roldan-Jimenez, et al. [23] confirmed these results when evaluating three sitting and standing up chair test conditions. In the first and second condition, there were 5 to 10 series respectively performed, both occurring at a rate of 40 beats per minute (controlled by a metronome). The last condition was the maximum repetition in 30 seconds. They observed that in the last condition, there was an increase in the muscular activation of the erector spine, and in all conditions the muscles that participated most in this sitting and standing up of the chair were tibialis anterior muscle (23-26\%), quadriceps (20-21\%), rectus abdominis muscle $(17-18 \%)$ and spine erectors (10\%). This finding shows that only with the overload of the body weight, there is a great contribution of the global muscles from 30 seconds of the action of sitting and standing up from the chair [23].

Regarding the importance of including measures of functional performance, the chair sitting and standing up test was identified as a predictor of mortality becoming an important functional measure in the elderly people, where each increase of one repetition is associated with a reduction of $21 \%$ in mortality [24]. We found only one previous study exploring the relationship between trunk muscle morphology (paraspinal muscles, abdominal and abdominal rectus muscles) and performance of functional tasks in healthy elderly people (70-79 years old). However, this study cannot explain the influence of the strength and endurance of the trunk muscles before functional activities [11].

On the other hand, although the functionality is more related to the endurance, the loss of strength of the trunk flexors is greater in the elderly people, when compared to the extensors, so it is also important to measure the maximum strength of this muscle group. Also, there is a marked loss in the cross-sectional area of the trunk muscles in the elderly population, which also influences the ability of the muscle to exert strength and consequently it can lead to spinal injuries [25].

Thus, it appears that the importance of trunk muscles for balance and mobility in the elderly people has been underestimated or perhaps neglected. Based on these results, it is important to improve the en- durance of the trunk muscles through specific interventions, perhaps contributing to an improvement in the functional performance of this population, with consequent reduction of falls and prevention of associated injuries in the lumbar region.

To our knowledge, this is the first study that analyzed the influence of endurance and trunk strength on variables that reflect functional activities of the elderly, such as removing a weight from the ground, stand up and walk, sit and stand up from a seat and take a walk. Thus, our results indicate that the maximum strength of the trunk will exert a greater participation in functional movements that require more of this variable, as is the case of performing maximum force to lift a weight from the ground, performing a triple extension of hip, knee and ankle. Otherwise, the trunk endurance will participate in functional movements that do not require the appendicular skeleton at maximum force. Nevertheless, the present study has the limitation of not being able to infer a cause and effect relationship between the elderly having a greater functionality and consequently having a greater endurance and/or trunk strength. Thus, clinical studies should be published in order to investigate this relationship.

\section{Conclusion}

We conclude that endurance of the extensor muscles of the trunk explains 17.9 to $24.4 \%$ of functional performance in trained elderly women. Thus, these results suggest that there is a great participation of the trunk muscles, mainly of the endurance, for activities that involve everyday actions.

\section{Data Availability}

The data used to support the results of this study are available for consultation, upon request to the corresponding author.

\section{Acknowledgments}

This study was financed in part by the Coordenação de Aperfeiçoamento de Pessoal de Nível Superior - Brasil (CAPES) - Finance Code 001. We are grateful for the hard work and dedication of the Functional Training Group (FTG) study group.

\section{Conflicts of Interest}

The authors declare that they have no conflicts of interest.

\section{Financial Support}

This study was financed in part by the Coordenação de Aperfeiçoamento de Pessoal de Nível Superior - Brasil (CAPES) - Finance Code 001.

\section{Authors Contributions}

M. M. A. M., conceptualization, data curation, formal analysis, investigation, methodology, writing - original 
draft, writing - review \& editing. M. S. S., A. B. S. V., A.G.R.N. and J.C.A.S., data curation, formal analysis, investigation, methodology, writing - original draft, writing - review \& editing. R. J. S. S., G. W. S. and P. M. P. O., data curation, formal analysis, investigation, methodology, writing - original draft, writing - review \& editing, project administration. F. J. A., E. H. M. D. and M. E. Da S-G, conceptualization, formal analysis, funding acquisition, project administration, resources, supervision, writing - original draft, writing - review \& editing.

\section{References}

1. Cadore EL, Rodríguez-Mañas L, Sinclair A, Izquierdo $M$ (2013) Effects of Different Exercise Interventions on Risk of Falls, Gait Ability, and Balance in Physically Frail Older Adults: A Systematic Review. Rejuvenation Res 16: 105114.

2. Cuellar WA, Wilson $A$, Blizzard CL, Otahal $P$, Callisaya ML, et al. (2017) The assessment of abdominal and multifidus muscles and their role in physical function in older adults: a systematic review. Physiotherapy 103: 21-39.

3. Kasukawa $\mathrm{Y}$, Miyakoshi N, Hongo M, Ishikawa $\mathrm{Y}$, Noguchi $H$, et al. (2010) Relationships between falls, spinal curvature, spinal mobility and back extensor strength in elderly people. J Bone Miner Metab 28: 82-87.

4. Paterson DH, Warburton DE (2010) Physical activity and functional limitations in older adults: a systematic review related to Canada's Physical Activity Guidelines. Int J Behav Nutr Phys Act 7: 38.

5. Wang CZ, Chen YJ, Wang YH, Yeh ML, Huang MH, et al. (2014) Low-level laser irradiation improves functional recovery and nerve regeneration in sciatic nerve crush rat injury model. PloS One 9: 103348.

6. Martien S, Delecluse C, Boen F, Seghers J, Pelssers J, et al. (2015) Is knee extension strength a better predictor of functional performance than handgrip strength among older adults in three different settings? Arch Gerontol Geriatr 60: 252-258.

7. Barbado D, Lopez-Valenciano A, Juan-Recio C, Montero-Carretero C, van Dieën JH, et al. (2016) Trunk Stability, Trunk Strength and Sport Performance Level in Judo. PLOS ONE 11: e0156267.

8. Kibler WB, Press J, Sciascia A (2006) The role of core stability in athletic function. Sports Med 36: 189-198.

9. Granacher U, Gollhofer A, Hortobágyi T, Kressig RW, Muehlbauer T (2013) The Importance of Trunk Muscle Strength for Balance, Functional Performance, and Fall Prevention in Seniors: A Systematic Review. Sports Med 43: 627-641.

10. Ebenbichler GR, Oddsson LI, Kollmitzer J, Erim Z (2001) Sensory-motor control of the lower back: implications for rehabilitation. Med Sci Sports Exerc 33: 1889-1898.

11. Hicks GE, Simonsick EM, Harris TB, Newman AB, Weiner DK, et al. (2005) Trunk muscle composition as a predictor of reduced functional capacity in the health, aging and body composition study: the moderating role of back pain. $J$ Gerontol A Biol Sci Med Sci 60: 1420-1424.
12. Behm DG, Drinkwater EJ, Willardson JM, Cowley PM (2010) The use of instability to train the core musculature. Appl Physiol Nutr Metab 35: 91-108.

13. Riuzzi F, Sorci G, Arcuri C, Giambanco I, Bellezza I, et al. (2018) Cellular and molecular mechanisms of sarcopenia: the S100B perspective: S100B and sarcopenia. J Cachexia Sarcopenia Muscle 9: 1255-1268.

14. Sutarno CG, McGill SM (1995) Isovelocity investigation of the lengthening behaviour of the erector spinae muscles. Eur J Appl Physiol Occup Physiol 70: 146-153.

15. Kienbacher T, Paul B, Habenicht R, Starek C, Wolf M, et al. (2014) Reliability of isometric trunk moment measurements in healthy persons over 50 years of age. J Rehabil Med 46: 241-249.

16. McGill SM, Childs A, Liebenson C (1999) Endurance times for low back stabilization exercises: clinical targets for testing and training from a normal database. Arch Phys Med Rehabil 80: 941-944.

17. Rikli RE, Jones CJ (2013) Development and Validation of Criterion-Referenced Clinically Relevant Fitness Standards for Maintaining Physical Independence in Later Years.Gerontologist 53: 255-267.

18. Chulvi-Medrano I, García-Massó X, Colado JC, Pablos C, de Moraes JA, et al. (2010) Deadlift Muscle Force and Activation Under Stable and Unstable Conditions: J Strength Cond Res 24: 2723-2730.

19. Shahtahmassebi B, Hebert JJ, Hecimovich MD, Fairchild TJ (2017) Associations between trunk muscle morphology, strength and function in older adults. Sci Rep 7.

20. Escamilla RF, Lewis C, Pecson A, Imamura R, Andrews JR (2016) Muscle Activation Among Supine, Prone, and Side Position Exercises With and Without a Swiss Ball. Sports Health 8: 372-379.

21. Suri P, Kiely DK, Leveille SG, Frontera WR, Bean JF (2011) Increased Trunk Extension Endurance Is Associated With Meaningful Improvement in Balance Among Older Adults With Mobility Problems. Arch Phys Med Rehabil 92: 10381043.

22. Leteneur S, Gillet C, Sadeghi H, Allard P, Barbier F (2009) Effect of trunk inclination on lower limb joint and lumbar moments in able men during the stance phase of gait. Clin Biomech (Bristol, Avon) 24: 190-195.

23. Roldán-Jiménez C, Bennett $P$, Cuesta-Vargas Al (2015) Muscular Activity and Fatigue in Lower-Limb and Trunk Muscles during Different Sit-To-Stand Tests. PLoS One 10: e0141675.

24. Brito LB, Ricardo DR, Araújo DS, Ramos PS, Myers J, et al. (2014) Ability to sit and rise from the floor as a predictor of all-cause mortality. Eur J Prev Cardiol 21: 892-898.

25. Sions JM, Elliott JM, Pohlig RT, Hicks GE (2017) Trunk Muscle Characteristics of the Multifidi, Erector Spinae, Psoas, and Quadratus Lumborum in Older Adults With and Without Chronic Low Back Pain. J Orthop Sports Phys Ther 47: 173-179. 